\title{
Whole Blood Lactate Kinetics in Patients Undergoing Quantitative Resuscitation for Severe Sepsis and Septic Shock
}

\author{
Michael A. Puskarich, MD; Stephen Trzeciak, MD; Nathan I. Shapiro, MD; \\ Andrew B. Albers, MD; Alan C. Heffner, MD; Jeffrey A. Kline, MD; and Alan E. Jones, MD
}

\begin{abstract}
Background: We sought to compare the association of whole-blood lactate kinetics with survival in patients with septic shock undergoing early quantitative resuscitation.

Methods: This was a preplanned analysis of a multicenter, ED-based, randomized, controlled trial of early sepsis resuscitation. Inclusion criteria were suspected infection, two or more systemic inflammation criteria, either systolic $\mathrm{BP}<90 \mathrm{~mm} \mathrm{Hg}$ after a fluid bolus or lactate level $>4 \mathrm{mM}$, two serial lactate measurements, and an initial lactate level $>2.0 \mathrm{mM}$. We calculated the relative lactate clearance, rate of lactate clearance, and occurrence of early lactate normalization (decline to $<2.0 \mathrm{mM}$ in the first $6 \mathrm{~h}$ ). Area under the receiver operating characteristic curve (AUC) and multivariate logistic regression were used to determine the lactate kinetic parameters that were the strongest predictors of survival.

Results: The analysis included 187 patients, of whom $36 \%(n=68)$ normalized their lactate level. Overall survival was $76.5 \%$ ( 143 of 187 patients), and the AUC of initial lactate to predict survival was 0.64. The AUCs for relative lactate clearance and lactate clearance rate were 0.67 and 0.58 , respectively. Lactate normalization was the strongest predictor of survival (adjusted OR, 5.2; 95\% CI, 1.7-15.8), followed by lactate clearance $\geq 50 \%$ (OR, 4.0; 95\% CI, 1.6-10.0). Lactate clearance $\geq 10 \%(\mathrm{OR}, 1.6 ; 95 \% \mathrm{CI}, 0.6-4.4)$ was not a significant independent predictor in this cohort. Conclusions: In patients in the ED with a sepsis diagnosis, early lactate normalization during the first $6 \mathrm{~h}$ of resuscitation was the strongest independent predictor of survival and was superior to other measures of lactate kinetics.
\end{abstract}

Trial registry: ClinicalTrials.gov; No.: NCT00372502; URL: clinicaltrials.gov

CHEST 2013; 143(6):1548-1553

Abbreviations: $\mathrm{IQR}=$ interquartile range; $\mathrm{ROC}=$ receiver operating characteristic; $\mathrm{ScvO}_{2}=$ central venous oxygen saturation; $\mathrm{SOFA}=$ sequential organ failure assessment

S

Cevere sepsis is a global health problem, resulting

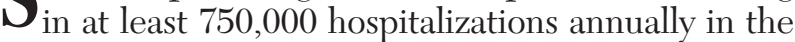
United States. ${ }^{1,2}$ Approximately 500,000 of these hospitalizations will be treated in US EDs. ${ }^{3}$ Early and aggressive quantitative resuscitative care is recommended for the treatment of septic shock ${ }^{4}$ and metaanalytic data have shown its efficacy at reducing mortality. ${ }^{5}$ However, presently there remains uncertainty about the optimal goals of early resuscitation, particularly regarding the roles of central venous oxygen saturation $\left(\mathrm{ScvO}_{2}\right)$ and lactate clearance. ${ }^{6,7}$

Elevated serum lactate measurements have been demonstrated to be independently associated with poor outcome in patients in the ED with infection. ${ }^{8}$ Previous investigations have documented that a lactate clearance of $10 \%$ during early sepsis resuscitation is independently associated with survival, a finding which persists even after adjusting for confounders. ${ }^{9,10}$ Previous work by our group found that in patients undergoing quantitative resuscitation for septic shock, achievement of a $\mathrm{ScvO}_{2} \geq 70 \%$ had a similar prog-

For editorial comment see page 1521

nosis as achievement of a lactate clearance of $10 \%$. However, failure to achieve a lactate clearance of $10 \%$ was associated with a mortality rate of $41 \%$ vs $8 \%$ if patients failed to achieve a $\mathrm{ScvO}_{2}$ of $70 \%$ (proportion difference 33\%, 95\% CI, 9\%-55\%). ${ }^{11}$ These data suggest that lactate clearance parameters may have better 
characteristics as resuscitation goals; however, it remains unclear which are the optimal lactate parameters to target during resuscitation. Accordingly, we sought to compare the relative value of various measures of whole-blood lactate kinetics to predict survival in patients with septic shock undergoing early quantitative resuscitation.

\section{Materials AND Methods}

\section{Study Design}

We conducted a preplanned analysis of a prospective, parallel group, nonblinded, randomized clinical trial designed to assess the noninferiority of lactate clearance $\mathrm{vs}^{\mathrm{ScvO}_{2}}$ as the protocol end point evaluating the adequacy of oxygen delivery during early sepsis resuscitation. ${ }^{12}$ The multicenter trial took place from January 2007 to January 2009 at Carolinas Medical Center, Charlotte, North Carolina; Beth Israel Deaconess Medical Center, Boston, Massachusetts; and Cooper University Hospital, Camden, New Jersey, all of which are large, urban, tertiary care hospitals staffed by emergency medicine resident physicians supervised by board-certified emergency medicine attending physicians. The study was approved by the institutional review board of Carolinas Healthcare System (09-06-02A), and all participants or their surrogates provided written informed consent for participation. The trial was registered on clinicatrials.gov, identifier NCT00372502.

The detailed methods of the study have been described previously. ${ }^{12}$ In brief, consecutive patients presenting to one of the participating EDs were eligible for enrollment if they were aged $>17$ years, had confirmed or suspected infection, two or more systemic inflammatory response criteria, ${ }^{13}$ and hypotension after fluid challenge or a blood lactate concentration of $\geq 4 \mathrm{mM}$. After enrollment, patients were randomly assigned to one of two groups. Each group received structured quantitative resuscitation while in the ED with iterative steps of IV crystalloid to achieve a central venous pressure $>8 \mathrm{~mm} \mathrm{Hg}$, followed by vasopressors to obtain a mean arterial pressure $>65 \mathrm{~mm} \mathrm{Hg}$, and finally packed RBC transfusion or administration of inotropes to achieve a $\mathrm{ScvO}_{2} \geq 70 \%$ or a lactate clearance of $10 \%$, depending on treatment group assignment. The study protocol was continued until all end points were achieved or for a maximum of $6 \mathrm{~h}$. The published results of this study showed a $6 \%(95 \% \mathrm{CI},-3 \%$ to $14 \%)$ in-hospital mortality difference favoring the lactate clearance group,

Manuscript received April 10, 2012; revision accepted October 12, 2012.

Affiliations: From the Department of Emergency Medicine (Drs Puskarich and Jones), University of Mississippi Medical Center, Jackson, MS; Departments of Medicine (Dr Trzeciak), Division of Critical Care Medicine and Emergency Medicine, Cooper University Hospital, Camden, NJ; Department of Emergency Medicine and Center for Vascular Biology Research (Dr Shapiro), Beth Israel Deaconess Medical Center, Boston, MA; and the Department of Emergency Medicine (Drs Albers, Heffner, and Kline), Carolinas Medical Center, Charlotte, NC.

Funding/Support: This work was supported by the National Institute of General Medical Sciences/National Institutes of Health [Grants HL091757, GM076659 (Dr Shapiro); R18HS01851901 (Dr Kline), K23GM076652; and GM083211 (Dr Jones)].

Correspondence to: Alan E. Jones, MD, Department of Emergency Medicine, University of Mississippi Medical Center, 2500 N State S, Jackson, MS, 39216; e-mail: aejones@umc.edu c) 2013 American College of Chest Physicians. Reproduction of this article is prohibited without written permission from the American College of Chest Physicians. See online for more details. DOI: 10.1378/chest.12-0878 confirming the primary hypothesis of noninferiority between the two resuscitation end points. ${ }^{12}$

As a part of the study protocol, research staff was instructed to perform blinded measurements of lactate clearance if patients were randomized to the $\mathrm{ScvO}_{2}$ arm. Blinded lactate measurements were performed by the research coordinator on a bedside pointof-care device, and results were printed onto a hard copy that was immediately gathered by the research coordinator. Measurements were performed using a deidentified patient identification number, which did not synchronize with the electronic medical record, and the treating clinicians had no way to access the data. At the suggestion of our hospital regulatory board, the simultaneous blinded measurements were encouraged, but not mandated, and, therefore, we expected they would not be performed on all subjects. Initial point-of-care lactate measurements were typically performed on the first blood draw, prior to any resuscitation except prehospital fluids. For the present study, we included patients with an abnormal initial lactate $(\geq 2 \mathrm{mM})$ who received at least two lactate measurements in the first $6 \mathrm{~h}$ of resuscitation in the ED.

\section{Data Analysis and Outcomes}

The primary outcome was in-hospital survival. Demographics and baseline characteristics of survivors were compared with those of nonsurvivors. Continuous data were compared using $t$ tests or Mann-Whitney tests, and categorical data were compared using $\chi^{2}$ or Fisher exact tests, as appropriate.

Normalization of lactate was defined as a lactate decline to $<2.0 \mathrm{mM}$. Absolute clearance (initial value minus delayed value), relative clearance (absolute clearance divided by initial value and the result multiplied by 100), and clearance rate (relative clearance divided by clearance time) were calculated. Receiver operating characteristic (ROC) curves were constructed for the ability of initial lactate, absolute clearance, relative clearance, and clearance rate to predict survival, and the area under the curve was calculated. Based on the ROC curves, lactate clearance cutoffs maximizing sensitivity and specificity were selected. Data were analyzed and ORs predicting survival between groups meeting and those failing to meet these various predefined lactate clearance parameters were calculated using Fisher exact tests with 95\% CI.

To account for potential confounders, a multivariate logistic regression model was created using in-hospital survival as the dependent variable. Candidate variables were selected based on known predictors of mortality and differences in survivors and nonsurvivors in the bivariate analysis, and the model was refined using reverse stepwise elimination. Adjusted ORs were calculated and are presented with $95 \%$ CIs. A post hoc subgroup analysis was performed in patients receiving catecholamine infusion vs patients who did not. Catecholamine infusion was defined as administration of dopamine or norepinephrine at any dose for any period of time while the patient was undergoing resuscitation in the ED and serial lactate measurements were being measured. As patients could have been enrolled in the study with hypotension but without an elevated lactate level, a portion of patients in our study had a lactate level between $2 \mathrm{mM}$ and $4 \mathrm{mM}$, thus, a post hoc analysis evaluating measures of lactate clearance was conducted in this subgroup as well. All statistical tests were two sided with $P<.05$ considered significant. Data were analyzed using commercially available statistical software (StatsDirect 2.7.7; StatsDirect Ltd and Stata 10.0; StataCorp LP).

\section{RESULTS}

Of 272 patients who had two serial lactate measurements, an initial lactate level $\geq 2 \mathrm{mM}$ was present in 187 (69\%), which composed the final study group. The 
overall survival in this group was 143 of 187 patients $(76.5 \%)$. Clinical and laboratory characteristics of the entire cohort, and specifically of survivors vs nonsurvivors, can be found in Table 1. Median initial lactate level was $4.4 \mathrm{mM}$ (interquartile range [IQR], 3.0-6.6), and the median absolute lactate clearance, median relative lactate clearance, and median relative lactate clearance rate were $1.9 \mathrm{mM}$ (IQR, 0.8-3.0 mM), 41\% (IQR, 19\%-61\%), and 18\% per hour (IQR, 9\%-25\%), respectively. Thirty-six percent of patients (68 of 187) normalized their lactate level during the first $6 \mathrm{~h}$ of resuscitation. The lactate clearance interventional arm comprised 98 of the 187 patients (52\%) included in the present analysis vs 89 patients in the control arm. There was no difference in mean or median number

Table 1-Patient Demographics and Clinical Characteristics

\begin{tabular}{|c|c|c|c|}
\hline Variable & $\begin{array}{l}\text { Survivors } \\
(\mathrm{n}=143)\end{array}$ & $\begin{array}{l}\text { Nonsurvivors } \\
\quad(\mathrm{n}=44)\end{array}$ & P Value \\
\hline Age, mean (SD), y & $60.0(16.7)$ & $67.0(13.7)$ & .01 \\
\hline \multicolumn{4}{|l|}{ Race } \\
\hline White & $75(52.4)$ & $27(61.4)$ & .30 \\
\hline Black American & $54(37.8)$ & $16(36.4)$ & .87 \\
\hline Hispanic & $13(9.0)$ & $0(0.0)$ & .03 \\
\hline Other & $1(0.7)$ & $1(2.2)$ & .42 \\
\hline Sex & & & .73 \\
\hline Male & $77(53.8)$ & $25(56.8)$ & \\
\hline Female & $66(46.2)$ & $19(43.2)$ & \\
\hline \multicolumn{4}{|l|}{ Preexisting conditions } \\
\hline Diabetes mellitus & $52(36.4)$ & $21(47.7)$ & .18 \\
\hline COPD & $26(18.1)$ & $9(20.5)$ & .83 \\
\hline End-stage renal disease & $16(11.2)$ & $5(11.4)$ & .99 \\
\hline Cirrhosis & $14(9.8)$ & $4(9.1)$ & .99 \\
\hline HIV-positive status & $13(9.1)$ & $2(4.6)$ & .53 \\
\hline \multicolumn{4}{|l|}{$\begin{array}{l}\text { Baseline laboratory values, } \\
\text { median (IQR) }\end{array}$} \\
\hline Hemoglobin, mg/dL & $11.3(9.8,13.4)$ & $11.6(9.9,13.8)$ & .66 \\
\hline Creatinine, mg/dL & $1.7(1.1,2.9)$ & $2.5(1.5,3.6)$ & .03 \\
\hline Total bilirubin, mg/dL & $1.1(0.6,1.8)$ & $0.8(0.7,1.4)$ & .33 \\
\hline Initial lactate level, mM & $4.3(3.0,6.1)$ & $5.9(3.4,8.3)$ & .007 \\
\hline \multicolumn{4}{|l|}{$\begin{array}{l}\text { Disease severity, } \\
\text { median }(\mathrm{IQR})^{\mathrm{b}}\end{array}$} \\
\hline SOFA score & $6(4,9)$ & $9.5(6,12)$ & $<.001$ \\
\hline \multicolumn{4}{|l|}{ Treatments } \\
\hline $\begin{array}{l}\text { Lactate clearance } \\
\text { protocol }\end{array}$ & $76(53.2)$ & $22(50.0)$ & .71 \\
\hline $\begin{array}{l}\text { IV fluids, }{ }^{\mathrm{c}, \mathrm{d}} \\
\text { median (IQR) }\end{array}$ & $4.0(2.5,5.5)$ & $4.3(3.0,5.0)$ & .54 \\
\hline Vasopressors & $86(60.1)$ & $31(70.5)$ & .22 \\
\hline Inotropes & $5(3.5)$ & $2(4.6)$ & .67 \\
\hline Transfusion of PRBCs & $7(4.9)$ & $2(4.6)$ & .99 \\
\hline Mechanical ventilation & $41(28.7)$ & $31(70.5)$ & $<.001$ \\
\hline Corticosteroids $^{\mathrm{d}}$ & $18(12.6)$ & $8(18.2)$ & .35 \\
\hline Activated protein $\mathrm{C}$ & $1(0.7)$ & $2(4.6)$ & .14 \\
\hline
\end{tabular}

Data given as total No. (\%) unless otherwise indicated. IQR $=$ interquartile range; $\mathrm{PRBC}=$ packed $\mathrm{RBC} ; \mathrm{SOFA}=$ Sequential Organ Failure Assessment.

aComparing survivors vs nonsurvivors.

bAt $0 \mathrm{~h}$.

In the first $6 \mathrm{~h}$

dAfter initial 2 L bolus. of lactate measurements between the two interventional arms in the parent study. Repeat lactate measurements were taken after a median of 133 min (IQR, 112-175 min) after the initial measurement.

Figure 1 shows the ROCs for initial lactate, relative lactate clearance, lactate clearance rate, and absolute lactate clearance. The area under the curve was highest for relative lactate clearance $(0.67)$ followed by initial lactate (0.64), lactate clearance rate (0.58), and absolute lactate clearance (0.56). Optimal relative lactate clearance based on the ROC curve to maximize the $\mathrm{OR}$ was $50 \%$. Absolute and relative clearance rate had poor predictive value, with optimal clearance of $0.1 \mathrm{mM}$ and $16 \%$ relative clearance per hour. Neither of these values was significantly associated with survival. Mortality rates in patients with lactate clearance rates of $5 \%, 10 \%, 15 \%, 20 \%, 25 \%$, and $30 \%$ per hour were similar: all between $18 \%$ to $21 \%$ without a clear trend. None of these rates demonstrated a statistically significant association with survival.

In the unadjusted analysis, lactate normalization was the best predictor of survival (OR, 6.3; 95\% CI, 2.4-17.0), followed by lactate clearance of $50 \%$ (OR, 4.3; 95\% CI, 1.8-10.2). Initial lactate value of 2 to $4 \mathrm{mM}$ (OR, 1.5; 95\% CI, 0.8-3.3) compared with $>4 \mathrm{mM}$ and lactate clearance of $10 \%$ were not statistically significant (OR, 1.7; 95\% CI, 0.8-4.0). The final multivariate model constructed to control for cofounding included age, initial Sequential Organ Failure Assessment (SOFA) score, and initial lactate, demonstrated good

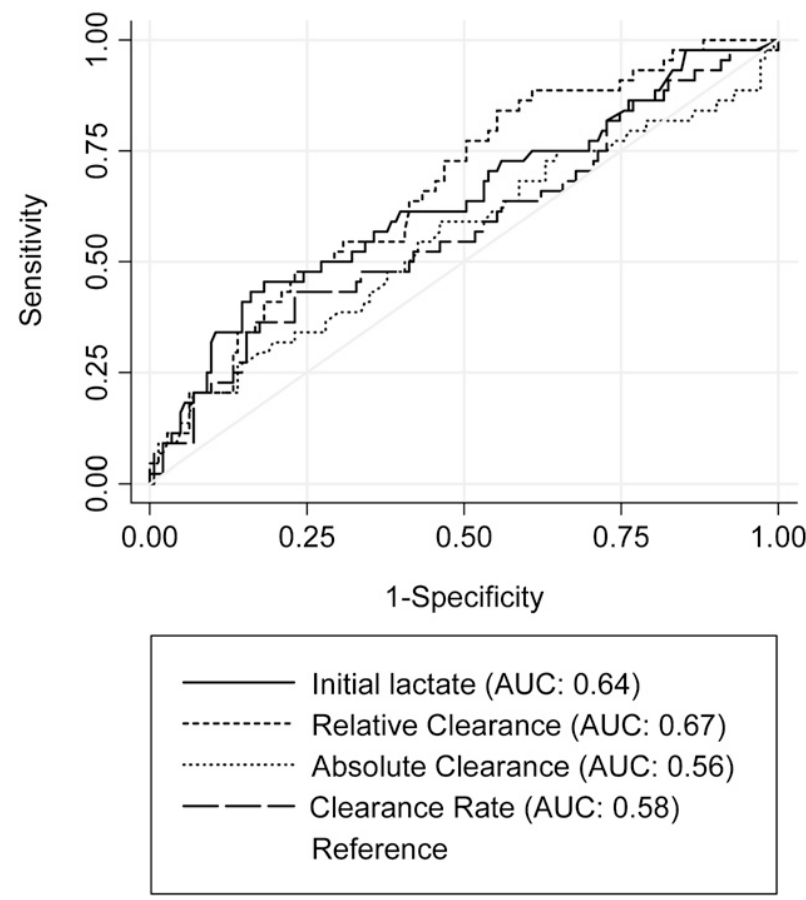

FiguRE 1. Receiver operator curves of initial lactate, absolute and relative lactate clearance, and lactate clearance rates in patients with an initial lactate $>2 \mathrm{mM}$. AUC $=$ area under the curve. 
calibration by the method of Hosmer-Lemeshow $(P=.35)$. While creatinine and mechanical ventilation were significantly different in the bivariate analysis, these measures are part of the SOFA score, and thus were dropped in the final model due to colinearity. When added individually to the model, relative lactate clearance of $50 \%$ and lactate normalization were significant independent predictors of survival $(P=.005$ and $P=.0045$, respectively). The adjusted ORs demonstrated no significant changes in the point estimates. The unadjusted and adjusted ORs are summarized in Table 2.

To assess for potential confounding by catecholamineinduced lactate production, the analysis was repeated after exclusion of patients receiving catecholamine vasopressors $(n=113)$. Only relative lactate clearance of $50 \%$ remained a statistically significant predictor of survival, probably due to loss of statistical power to detect a difference (data not shown). To assess for potential confounding by study allocation arm, the intervention (lactate clearance) arm was added to our multivariate analysis and demonstrated no significant impact on the model nor the resulting ORs.

As patients could be included in the study with hypotension but without a lactate level $>4 \mathrm{mM}$, this group was analyzed as a post hoc subgroup. This subgroup analysis demonstrated lower adjusted ORs for all studied measures of lactate clearance, with the same trend of strength of association that was found for the entire cohort (ie, lactate normalization was greater than relative lactate clearance of $50 \%$, which was greater than relative lactate clearance of $10 \%$ ). Lactate normalization and relative clearance of $50 \%$ remained statistically significant predictors of survival in this subgroup.

\section{DISCUSSION}

In this large, prospective study of early ED-based sepsis resuscitation, we obtained two subsequent whole-blood lactate measurements $2 \mathrm{~h}$ apart during early resuscitation and compared the association of various measures of lactate kinetics with in-hospital survival. To our knowledge, no study has directly compared various measures of lactate kinetics, including rate of lactate clearance and lactate normalization, in patients in the ED with septic shock undergoing early aggressive resuscitation. We found that normalization of lactate was a significant predictor of survival and was superior to other measures of lactate kinetics even after adjusting for potential confounders. Unsurprisingly, we found that nonsurvivors were significantly more likely to require mechanical ventilation, present with renal failure, and present with high initial lactate levels. However, even after adjustment for these potential confounders in our multivariate model, lactate normalization and relative lactate clearance of $50 \%$ were strong independent predictors of survival. These data suggest that lactate normalization may be an important resuscitation end point for investigation in future studies of sepsis resuscitation.

The prognostic value of initial lactate measurements in sepsis has been previously described. ${ }^{8,14,15}$ Additionally, delayed clearance of lactate in the initial days of ICU treatment of septic shock has been associated with poor outcomes, as demonstrated by Bakker et al. ${ }^{16}$ Nguyen et a ${ }^{9}$ demonstrated that early lactate clearance in patients in the ED undergoing early goal-directed therapy was associated with improved outcome, with a mean relative lactate clearance of $38 \%$ in survivors vs $12 \%$ in nonsurvivors. In their study, a relative lactate clearance of $10 \%$ was associated with the best combination of sensitivity and specificity for predicting survival after adjusting for other predictors of mortality, including initial lactate level. In contrast, we observed increasing survival among patients meeting higher relative lactate clearance parameters (specificity) without losing sensitivity (Table 2). We suspect one major reason for this difference may be the difference in initial lactate value: a mean baseline value of $6.9 \mathrm{mM}$ in the study by Nguyen et $\mathrm{al}^{9} \mathrm{vs}$ the median $4.4 \mathrm{mM}$ in our study. Additionally, Arnold et al ${ }^{10}$ confirmed the value of a relative lactate clearance of $10 \%$ in a multicenter study of ED patients undergoing resuscitation for severe sepsis, but did not evaluate other lactate clearance measurements, limiting comparisons to other lactate clearance parameters. Our findings enhance these previous reports by suggesting that both a relative lactate clearance of $50 \%$ and lactate normalization are more strongly associated with survival than previously reported clearance measures, including relative clearance of $10 \%$.

Table 2-Summary of Measures of Lactate Clearance in Patients: Association With Survival

\begin{tabular}{|c|c|c|c|c|c|c|c|}
\hline Variable & $\begin{array}{l}\text { Patients Meeting } \\
\text { Criteria }^{\mathrm{a}}\end{array}$ & $\begin{array}{l}\text { Survival of Those } \\
\text { Meeting Criteria }\end{array}$ & $\begin{array}{c}\text { Survival of Those Failing } \\
\text { to Meet Criteria }\end{array}$ & Unadjusted OR & $95 \% \mathrm{CI}$ & Adjusted OR & $95 \% \mathrm{CI}$ \\
\hline Lactate normalization & $68(36)$ & 93 & 67 & 6.3 & $2.4-17.0$ & 5.2 & $1.7-15.8$ \\
\hline Relative lactate clearance $50 \%$ & $71(38)$ & 90 & 68 & 4.3 & $1.8-10.2$ & 4.0 & $1.6-10.0$ \\
\hline Relative lactate clearance $10 \%$ & $159(85)$ & 78 & 68 & 1.7 & $0.8-4.0$ & 1.6 & $0.6-4.4$ \\
\hline Initial lactate level 2-4 mM & $83(44)$ & 81 & 73 & 1.5 & $0.8-3.1$ & 1.2 & $0.5-2.5$ \\
\hline
\end{tabular}

Data given as \%, unless otherwise indicated.

a Data given as total No. (\%). 
A study by Nichol et al ${ }^{17}$ suggested that timeweighted changes in lactate level in heterogeneous critical care patients in the first $24 \mathrm{~h}$ is predictive of survival. This study included all critically ill patients who received two lactate measurements over the first $24 \mathrm{~h}$ of their hospitalization. However, other published data suggest that lactate clearance may have different meaning in patients depending upon underlying pathology. This may be particularly true with patients with low oxygen transport states, where lactate clearance was not as predictive of outcome as in patients with sepsis. ${ }^{18}$ This difference between patient populations should not be surprising given the complexity of lactate production and metabolism in sepsis, influenced by both aerobic and anaerobic processes,${ }^{19}$ and the finding that quantitative resuscitation protocols only seem to offer benefit when initiated early. ${ }^{5}$ Furthermore, in hemodynamically stable patients with mild elevations in lactate level, these mild elevations appear to result primarily from decreased clearance rather than increased production. ${ }^{20}$ Given the variety of different causes for elevated lactate values, it may be inappropriate to compare lactate clearance in the hemodynamically stable patient in the ICU to those in the most proximate and acute phases of resuscitation. Thus, our data provide additional value by focusing on the acute phase of resuscitation of patients with severe sepsis and septic shock, and provide potentially important associative data for considering alternative resuscitation end points in early sepsis resuscitative care.

There continues to be scientific uncertainty about the optimal end points to target during early sepsis resuscitation. Recently, our group ${ }^{12}$ and others ${ }^{21}$ have shown that lactate clearance is a viable resuscitative end point. In the parent randomized control trial from which the present data are derived, relative lactate clearance of $10 \%$ was shown to be noninferior to $\mathrm{ScvO}_{2}$ as the final goal in early sepsis resuscitation. ${ }^{12}$ This goal was chosen based on a single study in the literature at the time comparing multiple levels of relative lactate clearance. ${ }^{9}$ The study by Jansen et al ${ }^{21}$ found that the addition of a relative lactate clearance goal of $20 \%$ improved survival in patients with septic shock, although in should be noted that these patients also received a comprehensive resuscitation strategy including $\mathrm{ScvO}_{2}$. In the present report, we extend our previous findings by investigating other parameters derived from serial lactate measurements during resuscitation. We found that lactate normalization had the strongest association with survival as compared with absolute clearance, relative clearance, and rate of clearance. We interpret these data to suggest that future experimental studies of sepsis resuscitation should investigate lactate normalization or higher levels of relative lactate clearance as resuscitation end points so that the relative benefits of driving additional lactate clearance with targeted therapies can be weighed against the risks of excessive fluid or vasoactive medication administration in the context of a well-designed clinical trial.

Interestingly, the rate of lactate clearance was not independently associated with survival, suggesting that the rapidity with which the lactate level is lowered is not as important as either the magnitude of clearance or achieving normalization within the first $6 \mathrm{~h}$ of resuscitation. However, while repeat lactate values were measured approximately $2 \mathrm{~h}$ apart (median $=133 \mathrm{~min}$; IQR, 112-175 min), limitations within the clinical trial prevented exact timing of repeat measurements. While lactate clearance rates were calculated based upon the number of minutes between measurements, it is possible that patients who had the repeat measurement done at a relatively later time had actually cleared their lactate some time earlier prior to measurement, leading to a measurement bias and underestimate of lactate clearance rate. Therefore, the authors urge caution in interpretation of clearance rate data and suggest this as a possible area of future investigation.

The strengths of this study are that patients had predefined serial lactate measurements during the initial phase of their resuscitation, and underwent early, aggressive, and prescribed resuscitation protocols. Multiple measures of lactate clearance were directly compared in the same patients, including previously unreported measures in this population of lactate clearance rate and lactate normalization.

Several weaknesses of this study deserve consideration as well. First, measures of lactate clearance are only useful in patients with hyperlactatemia. Second, lactate clearance was the final decision point in the resuscitation strategy to determine adequacy of oxygen delivery, and interventions targeting lactate clearance may have influenced our results in some patients, though such an interaction was not evident in our modeling. Third, this study was conducted at institutions with established ED-based quantitative resuscitation programs for sepsis prior to initiation of the study. Therefore, our results may not be generalizable to centers less experienced with early quantitative resuscitation. Fourth, patients were enrolled into one of two treatment protocols; however, assignment of treatment protocol did not differ between survivors and nonsurvivors, nor between patients who normalized and failed to normalize their lactate levels. Fifth, lactate measurements occurred approximately $2 \mathrm{~h}$ apart, and it is possible our calculated lactate clearance rates are an underestimate of the true value; thus, the value of lactate clearance rate remains an area for further investigation. Finally, based on our study design, we cannot rule out the influence of catecholamine-driven lactate production on the various measures of lactate clearances we studied. 


\section{ConClusions}

In patients with sepsis in the ED, undergoing early quantitative resuscitation, normalization of wholeblood lactate during resuscitation was more strongly associated with survival than any absolute value or absolute/relative change in lactate levels. Future studies should address if strategies targeting lactate normalization leads to improved outcomes.

\section{ACKNOWLEDGMENTS}

Author contributions: Dr Jones had full access to all of the data in the study and takes responsibility for the integrity of the data and the accuracy of the data analysis.

Dr Puskarich: contributed to data analysis, drafting and revision of the manuscript, and approval of the final manuscript and served as principal author.

Dr Trzeciak: contributed to the study design, data collection, revision of the manuscript, and approval of the final manuscript.

Dr Shapiro: contributed to the study design, data collection, revision of the manuscript, and approval of the final manuscript.

Dr Albers: contributed to data collection, revision of the manuscript, and approval of the final manuscript.

Dr Heffner: contributed to data collection, revision of the manuscript, and approval of the final manuscript.

Dr Kline: contributed to the study design, revision of the manuscript, and approval of the final manuscript.

Dr Jones: conceived the study, contributed to the study design, data collection and analysis, drafting revision of the manuscript, and approval of the final version of the manuscript.

Financial/nonfinancial disclosures: The authors have reported to CHEST that no potential conflicts of interest exist with any companies/organizations whose products or services may be discussed in this article.

Role of sponsors: The sponsor had no role in the design, implementation, or analysis of the study and had no input into the writing of the manuscript.

\section{REFERENCES}

1. Angus DC, Linde-Zwirble WT, Lidicker J, Clermont G, Carcillo J, Pinsky MR. Epidemiology of severe sepsis in the United States: analysis of incidence, outcome, and associated costs of care. Crit Care Med. 2001;29(7):1303-1310.

2. Dombrovskiy VY, Martin AA, Sunderram J, Paz HL. Rapid increase in hospitalization and mortality rates for severe sepsis in the United States: a trend analysis from 1993 to 2003. Crit Care Med. 2007;35(5):1244-1250.

3. Wang HE, Shapiro NI, Angus DC, Yealy DM. National estimates of severe sepsis in United States emergency departments. Crit Care Med. 2007;35(8):1928-1936.

4. Dellinger RP, Levy MM, Carlet JM, et al; International Surviving Sepsis Campaign Guidelines Committee; American Association of Critical-Care Nurses; American College of Chest Physicians; American College of Emergency Physicians; Canadian Critical Care Society; European Society of Clinical Microbiology and Infectious Diseases; European Society of Intensive Care Medicine; European Respiratory Society; International Sepsis Forum; Japanese Association for Acute Medicine; Japanese Society of Intensive Care Medicine; Society of Critical Care Medicine; Society of Hospital Medicine; Surgical Infection Society; World Federation of Societies of Intensive and Critical Care Medicine. Surviving sepsis campaign: international guidelines for management of severe sepsis and septic shock: 2008. Crit Care Med. 2008;36(1): 296-327.

5. Jones AE, Brown MD, Trzeciak S, et al; Emergency Medicine Shock Research Network investigators. The effect of a quantitative resuscitation strategy on mortality in patients with sepsis: a meta-analysis. Crit Care Med. 2008;36(10):2734-2739.

6. Jones AE. Point: should lactate clearance be substituted for central venous oxygen saturation as goals of early severe sepsis and septic shock therapy? Yes. Chest. 2011;140(6):1406-1408.

7. Rivers EP, Elkin R, Cannon CM. Counterpoint: should lactate clearance be substituted for central venous oxygen saturation as goals of early severe sepsis and septic shock therapy? No. Chest. 2011;140(6):1408-1413.

8. Shapiro NI, Howell MD, Talmor D, et al. Serum lactate as a predictor of mortality in emergency department patients with infection. Ann Emerg Med. 2005;45(5):524-528.

9. Nguyen HB, Rivers EP, Knoblich BP, et al. Early lactate clearance is associated with improved outcome in severe sepsis and septic shock. Crit Care Med. 2004;32(8):1637-1642.

10. Arnold RC, Shapiro NI, Jones AE, et al. Multi-center study of early lactate clearance as a determinant of survival in patients with presumed sepsis. Shock. 2009;32(1):35-39.

11. Puskarich MA, Trzeciak S, Shapiro NI, et al; Emergency Medicine Shock Research Network (EMSHOCKNET). Prognostic value and agreement of achieving lactate clearance or central venous oxygen saturation goals during early sepsis resuscitation. Acad Emerg Med. 2012;19(3):252-258.

12. Jones AE, Shapiro NI, Trzeciak S, Arnold RC, Claremont HA, Kline JA; Emergency Medicine Shock Research Network (EMShockNet) Investigators. Lactate clearance vs central venous oxygen saturation as goals of early sepsis therapy: a randomized clinical trial. JAMA. 2010;303(8):739-746.

13. Bone RC, Balk RA, Cerra FB, et al; The ACCP/SCCM Consensus Conference Committee. American College of Chest Physicians/Society of Critical Care Medicine. Definitions for sepsis and organ failure and guidelines for the use of innovative therapies in sepsis. Chest. 1992;101(6):1644-1655.

14. Mikkelsen ME, Miltiades AN, Gaieski DF, et al. Serum lactate is associated with mortality in severe sepsis independent of organ failure and shock. Crit Care Med. 2009;37(5):1670-1677.

15. Trzeciak S, Dellinger RP, Chansky ME, et al. Serum lactate as a predictor of mortality in patients with infection. Intensive Care Med. 2007;33(6):970-977.

16. Bakker J, Gris P, Coffernils M, Kahn RJ, Vincent JL. Serial blood lactate levels can predict the development of multiple organ failure following septic shock. Am J Surg. 1996;171(2):221-226.

17. Nichol A, Bailey M, Egi M, et al. Dynamic lactate indices as predictors of outcome in critically ill patients. Crit Care. 2011; 15(5):R242.

18. Jansen TC, van Bommel J, Mulder PG, et al. Prognostic value of blood lactate levels: does the clinical diagnosis at admission matter? J Trauma. 2009;66(2):377-385.

19. Jansen TC, van Bommel J, Bakker J. Blood lactate monitoring in critically ill patients: a systematic health technology assessment. Crit Care Med. 2009;37(10):2827-2839.

20. Levraut J, Ciebiera JP, Chave S, et al. Mild hyperlactatemia in stable septic patients is due to impaired lactate clearance rather than overproduction. Am J Respir Crit Care Med. 1998;157(4 pt 1):1021-1026.

21. Jansen TC, van Bommel J, Schoonderbeek FJ, et al; LACTATE study group. Early lactate-guided therapy in intensive care unit patients: a multicenter, open-label, randomized controlled trial. Am J Respir Crit Care Med. 2010;182(6):752-761. 